\title{
Cerebral perfusion in chronic fatigue syndrome
} and depression

\author{
SIOBHAN M. MACHALE, STEPHEN M. LAWRIE, JONATHAN T. O. CAVANAGH, \\ MIKE F. GLABUS, CATHERINE L. MURRAY, GUY M. GOODWIN and \\ KLAUS P. EBMEIER
}

\begin{abstract}
Background Patients with chronic fatigue syndrome (CFS) and depressive illness share many, but not all, features.
\end{abstract}

\begin{abstract}
Aims To test the hypothesis that patients with CFS have abnormal cerebral perfusion, that differs from that in patients with depressive illness.
\end{abstract}

Method We recruited 30 patients with CFS who were not depressed, 12 depressed patients and 15 healthy volunteers. Regional cerebral perfusion at rest was assessed using region of interest (ROI) and voxel-based statistical parametric mapping (SPM) techniques.

\section{Results On SPM analysis there was} increased perfusion in the right thalamus, pallidum and putamen in patients with CFS and in those with depressive illness. CFS patients also had increased perfusion in the left thalamus. Depressed patients differed from those with CFS in having relatively less perfusion of the left prefrontal cortex. The results were similar on ROI analysis.

\section{Conclusions Abnormal cerebral perfusion patterns in CFS subjects who are not depressed are similar but not identical to those in patients with depressive illness. Thalamic overactivity may be a correlate of increased attention to activity in CFS and depression; reduced prefrontal perfusion in depression may be associated with the greater neuropsychological deficits in that disorder.}

\section{Declaration of interest Study} funded by the Scottish Home and Health Department and the Wellcome Trust.
Chronic fatigue syndrome (CFS) is characterised by incapacitating fatigue of at least 6 months' duration, that is made worse by relatively minor exertion. Although depressive or anxiety disorders have been diagnosed in up to two-thirds of CFS sufferers, the nature of the relationship between CFS and depression remains uncertain (Kendell, 1991). One way to identify similarities and differences between CFS and depressive illness is to study their biological correlates through the use of neuroimaging techniques. Functional imaging studies have yielded inconsistent results to date. Most of the single-photon emission computerised tomography (SPECT) studies have reported areas of cerebral hypoperfusion, either global (Ichise et al, 1992; Schwartz et al, 1994) or localised (Costa et al, 1995; Goldstein et al, 1995). However, others have reported no significant cerebral perfusion differences compared with healthy (Peterson et al, 1994) and/or depressed (Fischler et al, 1996) controls. These inconsistencies may be explained in terms of (a) patient selection (as most of the studies have not explicitly excluded confounding psychopathology), and (b) different scanning methodology - most studies to date have used relatively subjective, non-standardised techniques for reporting statistical abnormalities of cerebral perfusion. In this paper, we present a comparison between patients with CFS who do not have a concurrent depressive illness, patients with major depressive disorders (both groups diagnosed with standardised criteria) and healthy volunteers. The results are from high-resolution single-photon emission tomography, with both region of interest (ROI) and voxel-based statistical parametric mapping (SPM) analysis of the effects of diagnosis on perfusion.

\section{METHOD}

\section{Chronic fatigue syndrome}

Thirty ambulant subjects with CFS were recruited from a local infectious diseases unit
(17 subjects) and a local self-help group (13 subjects). We screened a further seven people from the infectious diseases unit and 63 from the self-help group - the majority of the latter were excluded as they did not meet CFS criteria and/or were not interested in the study; most of the exclusions from the former group were due to scoring above case threshold on the Hospital Anxiety and Depression Scale (HAD). All participants had a thorough clinical evaluation, including physical examination and laboratory screening tests as recommended by the International CFS Study Group (Fukuda et al, 1994). The HAD questionnaire (Zigmond \& Snaith, 1983) and a standardised psychiatric interview (Schedule for Affective Disorders and Schizophrenia: SADS) (Endicott \& Spitzer, 1978) were used to exclude subjects with current psychiatric illness, with a particular emphasis on depression. Subjects with depression or anxiety disorders were excluded, but the one with somatisation disorder was included. The Hamilton Depression Rating Scale (HDRS) was used as a measure of severity of any depressive symptoms (Hamilton, 1960). The mean duration of illness was 68 months (range 6-240). Sixteen subjects were medicationfree at the time of imaging; the remaining 14 had been prescribed either antidepressants (10), minor tranquillisers (4), and/or endocrine replacement (two on thyroxine, one on oral contraceptive). Of the $10 \mathrm{pa}$ tients prescribed antidepressants, five had a previous diagnosis of depression, of whom three were still receiving full antidepressant doses. The remaining five patients were prescribed antidepressant medication for symptomatic relief, generally 25$50 \mathrm{mg}$ of a tricyclic antidepressant (or its equivalent). Patients were excluded if there had been any changes in their medication in the preceding 3 weeks. Current fatigue levels on the day of testing were rated using the fatigue scale of the health scale (Befindlichkeitsskala) of von Zerssen et al (1974). Subjects within the CFS and healthy volunteer groups also completed the LikertChalder Fatigue Scale (Chalder et al, 1993), which rates levels of fatigue over the preceding month. Demographic data and clinical scores are summarised in Table 1.

\section{Depressed patients}

Twelve hospital in-patients with a diagnosis of major depressive episode with 
melancholia and no history of (hypo-) mania (DSM-III-R; American Psychiatric Association, 1987) were recruited. The mean duration of their present episode was 12 months (range 2-64). Two patients were medication-free at the time of imaging. Of the remaining 10 , three were prescribed neuroleptics, two lithium carbonate, eight antidepressants, three hypnotics or anxiolytics, and one endocrine replacement (thyroxine). Patients were excluded if there had been any changes in their drug regime in the previous 3 weeks. Five patients had received electroconvulsive therapy (ECT) more than 6 months ago. Patients had two scans within 24 hours, and data on diurnal variations of mood in this group have been reported previously (Ebmeier et al, 1997). The morning scan was chosen for all depressed patients, and only patients randomised into the 'morning first' group were included, to make scanning times comparable with those of the other groups.

\section{Healthy volunteers}

Fifteen healthy volunteers, who were matched in aggregate for age and gender, were recruited as a control group from hospital staff and friends of patients. Any significant medical and psychiatric conditions were excluded by thorough evaluation, as outlined above, including screening blood tests and psychiatric interview. Three subjects were receiving regular medication in the form of hormone replacement therapy or oral contraception.

The study followed protocols approved by the local ethics committee and the Administration of Radioactive Substances
Advisory Committee (ARSAC) at the UK Department of Health.

\section{Imaging protocol}

All subjects were imaged with a single-slice 12-detector head scanner with an in-slice and $z$-axis resolution of $8.5 \mathrm{~mm}$ full-width half-maximum (FWHM) and a sensitivity of 15000 counts $/ \mathrm{s} / \mathrm{mCi} / \mathrm{ml}$, using the intermediate 572-hole collimators (Neuro 900, Strichman Medical Equipment Inc., Boston, USA). Healthy volunteers and patients with depression received $250 \mathrm{MBq}$ as part of a split-dose scanning procedure (Ebmeier $e t$ al, 1991, 1997). CFS patients received $500 \mathrm{MBq}$ of technetium-99m hexamethyl propylamineoxime ( $\left.{ }^{99 \mathrm{~m}} \mathrm{Tc}-\mathrm{HM}-\mathrm{PAO}\right)$. The scanning time for the first two groups was doubled to $5 \mathrm{~min}$ per slice in order to compensate for the reduced dose. Data on the satisfactory reliability of half-dose as compared with full-dose scans have been published previously (Ebmeier et al, 1991).

Subjects rested comfortably on the imaging table with eyes closed and covered and environmental noise kept to a minimum, while the tracer injection was administered over a $30 \mathrm{~s}$ period. The subject's head was then positioned in a moulded head-holder and aligned with the help of two crossed positioning lights. During the scan, the head was fixed with pressure pads over the zygomatic arches. Slices were acquired parallel to the orbito-meatal plane, starting at a level approximately $2 \mathrm{~cm}$ above the orbito-meatal line and at $1 \mathrm{~cm}$ intervals above this level; further details of the method have been described previously (Ebmeier et al, 1995). Images were reconstructed using software supplied by Strichmann Medical Equipment (SME) for the Apple Macintosh. The SME reconstruction algorithm selects an enveloping ellipse, derived from an oversmoothed image of the brain. This, together with the absorption length parameter $(95 \mathrm{~mm})$, determines the Chang-like attention correction which was done with one iteration. Count distributions were deconvoluted into the radioisotope concentrations responsible, using a Wiener filter with a correlation length of $6 \mathrm{~mm}$.

\section{Image analysis}

Two transverse slices were chosen for the ROI analysis, approximately 4 and $6 \mathrm{~cm}$ above the orbito-meatal line. A standard template was prepared by drawing regions of interest over corresponding brain atlas slices (Talairach et al, 1988). Although this atlas is oriented to an internal anatomical reference (the line between anterior and posterior commissures), the orbito-meatal line is almost parallel with it (Szikla et al, 1977). The ROIs included, in the lower slice, frontal, anterior and posterior cingulate, anterior temporal, posterior temporal, calcarine and occipital cortex, as well as caudate, putamen and thalamus. The corresponding template for the higher slice contained frontal, anterior and posterior cingulate, parietal and occipital cortex. The templates are linearly and symmetrically deformed to fit different brain sizes and shapes, using the $20 \%$ isocontour line to define the cortical edge. ROI were thus preserved in their relative position to each other, and no additional adjustments were made for single regions that appeared to be

Table I Descriptive and clinical characteristics of patient and control groups

\begin{tabular}{|c|c|c|c|c|c|}
\hline Variable & $\begin{array}{l}\text { Chronic fatigue } \\
\text { syndrome }\end{array}$ & Depression & $\begin{array}{l}\text { Healthy } \\
\text { volunteers }\end{array}$ & $\begin{array}{l}\text { Test and overall } \\
\text { significance }\end{array}$ & Post hoc significance \\
\hline Mean age, years (s.d.) & $44.2(10.3)$ & $44.3(12.5)$ & $4 I . I(12.8)$ & $F=0.33, P=0.71$ & \\
\hline Gender (male:female) & 11:19 & $6: 6$ & $4: 11$ & Pearson $\chi^{2}=1.56, P=0.46$ & \\
\hline Mean (s.d.) $I^{\prime}$ & $114.2(10.3)$ & $108.0(10.2)$ & $113(9.5)$ & $\mathrm{F}=1.53, P=0.23$ & \\
\hline $\begin{array}{l}\text { Median fatigue on Befindlichskeitsskala (inter-quartile } \\
\text { range) }\end{array}$ & II.0 (6.0-14.0) & $13.0(5.0-14.0)$ & $0.0(0.0-3.0)$ & $\begin{array}{l}\text { Kruskal-Wallis } \\
\chi^{2}=15.5, P=0.004\end{array}$ & CFS, Dep > HV \\
\hline Mean Likert-Chalder fatigue (s.d.) & $27.1(3.6)$ & - & II.8 (I.5) & $\mathrm{F}=252.8, P=0.000 \mathrm{I}$ & \\
\hline Median Hamilton Depression Scale (inter-quartile range) & $8.5(7.0-11.0)$ & $24.0(20.5-27.5)$ & $0.0(0.0-1.0)$ & $\begin{array}{c}\text { Kruskal-Wallis } \\
\chi^{2}=46.6, P=0.00 \mathrm{I}\end{array}$ & Dep $>$ CFS $>$ HV \\
\hline
\end{tabular}

I. IQ measured on National Adult Reading Test (NART); for references to other variables employed, see text.

CFS, chronic fatigue syndrome; Dep, depression; HV, healthy volunteers. 
out of position (Ebmeier et al, 1991). Regional count densities were normalised by proportional scaling to whole brain blood flow (derived from the two slices examined). The reliability of this ROI method has been examined previously, in both control and patient groups, with between-rater errors approximating 10\% (Ebmeier et al, 1991).

Images were further processed using Analyze (CNS Software) with customwritten software developed in the MATLAB (The Mathworks, Inc.) on a Sun SPARC workstation. SPM of regional cerebral tracer uptake were derived using the SPM96 software supplied by K. J. Friston and colleagues at the Wellcome Department of Cognitive Neurology. The SPM data were processed as follows:

(a) images were exported as binary files and converted to Analyze format;

(b) the scans were spatially normalised using a 12-point linear affine transformation into Talairach space (Friston $e t$ al, 1995);

(c) the images were then smoothed with an isotropic (12 mm FWHM) Gaussian filter to reduce the error variance associated with individual variability in gyral anatomy, to improve the signalto-noise ratio, and to precondition the data to fit the Gaussian distribution more closely;

(d) differences in global cerebral tracer activity between subjects were removed by proportional scaling, similar to the ROI procedure.

\section{Statistics}

Significant group effects are reported for the ROI analysis using analysis of variance (ANOVA) followed by post hoc Scheffetests $(P<0.05)$, and for the SPM analysis using peak $z$-values with $P$-values corrected for multiple comparisons. SPM significance at cluster level is based on simultaneous consideration of: (a) the peak effect size within a contiguous volume with voxel $z$-values greater than 2.33; (b) the size of this volume; (c) the smoothness of the data-set; and (d) the overall size of the search volume. Only regions significant at both voxel and cluster level were accepted. Data were analysed using an analysis of covariance (ANCOVA) design with age as a confounding covariate. In order to exclude medication effects, a subgroup analysis was performed, removing the CFS patients who were prescribed psychotropic medications. As this did not alter the findings of statistical significance, the result for the whole group is reported.

\section{RESULTS}

Clinical and demographic data for all three groups are presented in Table 1. All subjects were Caucasian, and the groups were well matched in aggregate for age, gender, premorbid IQ (National Adult Reading Test; Nelson, 1982) and handedness. Healthy volunteers were clearly less fatigued than the patient groups. Median $\mathrm{Ha}$ milton Depression scores were highest for the depressed patients, significantly lower in the CFS patients, and lowest in healthy volunteers.

Table 2 lists the peak differences in perfusion between the three diagnostic groups using SPM. Figure 1 illustrates perfusion differences between patient groups and healthy volunteers in a slice $40 \mathrm{~mm}$ above the orbitomeatal line. Uptake was greater, mainly in the right thalamus (as well as pallidum and putamen), both in subjects with CFS and those with depression, than in healthy controls. CFS patients also showed increased perfusion in the left thalamus. In comparison with the CFS group, in the patients with depression perfusion was decreased in the left prefrontal cortex. The remaining foci in Fig. 1 did not achieve statistical significance at the cluster (volume) level.

The results of the ROI analysis are shown in Table 3. The pattern of results in the thalamus and putamen is similar to the patterns obtained with SPM, although the differences are non-significant, apart from in the left thalamus and right caudate and putamen in unmedicated CFS patients. The significant ROI differences in caudate nuclei could be due to the cortical rim fitting of the template, which can result in partial volume effects between central structures and ventricular cerebrospinal fluid.

\section{DISCUSSION}

\section{Main findings}

We found that CFS and depression are associated with increased blood flow in the right thalamus. In support of previous ROI studies (Goldstein et al, 1995; Fischler et al, 1996) we found little difference between the two groups, other than the pattern of perfusion in the left prefrontal cortex on SPM analysis. This is in keeping with previous reports of decreased left prefrontal perfusion in depression (Goodwin, 1997). In agreement with the work of Fischler et al (1996) which, like our own, normalised regional activity to total brain perfusion, we did not replicate reports of global or localised cerebral hypoperfusion in CFS. We believe that this is most likely to be explained by the fact that our CFS patients had been carefully screened for comorbid psychiatric disorders (especially depression) and were well matched for potential confounders, as well as by our use of the more robust whole-brain normalisation of cerebral perfusion. We were not able to address the possibility of reduced perfusion of the brain-stem (Costa et al, 1995), mainly because of the lack of reliable data from an area whose size lies at the spatial resolution limit of SPECT.

\section{Limitations}

The main limitation of the present study is that our CFS subjects had high levels of

Table 2 Effect of diagnosis on cerebral perfusion (statistical parametric mapping analysis). All areas containing voxels with uncorrected $P<0.00 \mathrm{I}$ are listed

\begin{tabular}{|c|c|c|c|c|c|}
\hline Effect & Region of increased perfusion & $z$-Value & Volume $\left(\mathrm{cm}^{3}\right)$ & Peak coordinates & Corrected $P$-value \\
\hline \multirow[t]{2}{*}{ CFS v. healthy volunteers } & Right thalamus, pallidum and putamen & 5.35 & 10.6 & $14,-4,0$ & 0.002 \\
\hline & Left thalamus & 4.56 & & $-16,-22,4$ & 0.017 \\
\hline Depression $v$. healthy volunteers & Right thalamus, pallidum and putamen & 5.02 & 16.3 & $22,-8,0$ & 0.009 \\
\hline CFS v. depression & Left prefrontal cortex & 4.38 & 11.3 & $-34,46,16$ & 0.023 \\
\hline
\end{tabular}

CFS, chronic fatigue syndrome. 
depression: almost half were on psychotropic medication and five had a previous history of depression. This reflects the rarity of CFS without comorbid psychiatric disturbance, and is only evident because we characterised our subjects so carefully. Our rigorous screening and inclusion of self-help group members may, of course, mean that our CFS subjects are not representative of people with CFS in clinics and in the community. Almost all our depressed subjects were medicated, and psy- chotropic medication may well alter cerebral perfusion, although the findings in our CFS subjects do not appear to be explained by medication effects. It should also be noted that our controls were recruited as staff or friends and may not therefore be representative of the 'normal' population.

\section{Methodological issues}

This is the first study to report SPM analysis of the pattern of cerebral perfusion in patients with CFS compared with wellmatched groups of depressed and normal controls. SPM was originally used in positron emission tomography (PET) and has only recently been applied to SPECT data. As the previous SPECT studies in this area have used ROI analysis, we report both ROI results and the SPM analysis for comparison and validation. The trends shown in the ROI analysis were generally in agreement with the SPM results. ROI analysis is potentially prone to human error, as the

Table 3 Effect of diagnosis on regional perfusion (region of interest analysis)

\begin{tabular}{|c|c|c|c|c|c|c|c|c|}
\hline & \multicolumn{2}{|c|}{$\begin{array}{l}\text { Healthy volunteers } \\
\qquad n=15\end{array}$} & \multicolumn{2}{|c|}{$\begin{array}{l}\text { Chronic fatigue syndrome } \\
\qquad \begin{array}{l}n=30 \\
\end{array}\end{array}$} & \multicolumn{2}{|c|}{$\begin{array}{c}\text { Depression (a.m.) } \\
\qquad n=12\end{array}$} & \multicolumn{2}{|c|}{ One-way ANOVA } \\
\hline & Mean & s.d. & Mean & s.d. & Mean & s.d. & $P\left(F_{2}, 54\right)$ & Scheffé $(P<0.05)$ \\
\hline \multicolumn{9}{|l|}{ Left } \\
\hline Frontal & 1.06 & 0.03 & 1.06 & 0.05 & 1.03 & 0.06 & 0.23 & \\
\hline Anterior temporal & 1.12 & 0.07 & 1.12 & 0.07 & 1.10 & 0.10 & 0.36 & \\
\hline Posterior temporal & 1.13 & 0.04 & 1.13 & 0.04 & I.II & 0.07 & 0.48 & \\
\hline Occipital & 1.10 & 0.06 & 1.09 & 0.06 & 1.09 & 0.09 & 0.97 & \\
\hline Calcarine & 1.14 & 0.09 & 1.18 & 0.09 & 1.20 & 0.07 & 0.17 & \\
\hline Anterior cingulate & 1.15 & 0.09 & 1.08 & 0.10 & 1.09 & 0.11 & 0.07 & \\
\hline Posterior cingulate & 0.94 & 0.10 & 0.90 & 0.09 & 0.87 & 0.12 & 0.18 & \\
\hline Caudate & 1.19 & 0.15 & 1.23 & 0.06 & 0.98 & 0.16 & 0.0000 & $H V, F>D$ \\
\hline Putamen & 1.27 & 0.08 & 1.29 & 0.08 & 1.24 & 0.10 & 0.13 & \\
\hline Thalamus & 1.24 & 0.08 & $1.27^{2}$ & 0.06 & 1.24 & 0.09 & 0.28 & \\
\hline Frontal' & 1.05 & 0.03 & 1.04 & 0.04 & 1.03 & 0.03 & 0.25 & \\
\hline Parietal' & 1.07 & 0.03 & $\mathrm{I} .08$ & 0.04 & 1.08 & 0.07 & 0.66 & \\
\hline Occipital' & 1.12 & 0.04 & 1.14 & 0.06 & $\mathrm{I} .14$ & 0.08 & 0.35 & \\
\hline Anterior cingulate ${ }^{\prime}$ & 1.24 & 0.08 & 1.25 & 0.09 & 1.27 & 0.09 & 0.77 & \\
\hline Posterior cingulate' & 1.30 & 0.06 & $\mathrm{I} .34$ & 0.10 & 1.33 & 0.10 & 0.51 & \\
\hline \multicolumn{9}{|l|}{ Right } \\
\hline Frontal & 1.06 & 0.04 & $\mathrm{I} .07$ & 0.05 & 1.05 & 0.06 & 0.26 & \\
\hline Anterior temporal & 1.15 & 0.06 & 1.12 & 0.06 & 1.15 & 0.07 & 0.11 & \\
\hline Posterior temporal & 1.10 & 0.04 & 1.12 & 0.05 & I.II & 0.07 & 0.45 & \\
\hline Occipital & 1.05 & 0.06 & 1.08 & 0.06 & 1.08 & 0.11 & 0.49 & \\
\hline Calcarine & 1.20 & 0.09 & 1.18 & 0.09 & 1.16 & 0.08 & 0.53 & \\
\hline Anterior cingulate & 1.10 & 0.08 & 1.09 & 0.09 & 1.07 & 0.10 & 0.62 & \\
\hline Posterior cingulate & 1.00 & 0.06 & 0.98 & 0.09 & 0.93 & 0.14 & 0.15 & \\
\hline Caudate & 1.16 & 0.11 & $\mathrm{I} .2 \mathrm{I} *$ & 0.06 & 0.96 & 0.19 & 0.0000 & $H V, F>D$ \\
\hline Putamen & 1.26 & 0.08 & $1.30 *$ & 0.07 & 1.24 & 0.09 & 0.03 & $\dagger$ \\
\hline Thalamus & $\mathrm{I} .2 \mathrm{I}$ & 0.07 & 1.26 & 0.06 & 1.26 & 0.10 & 0.08 & \\
\hline Frontal' & $\mathrm{I} .04$ & 0.03 & 1.05 & 0.03 & 1.05 & 0.03 & 0.61 & \\
\hline Parietal' & 1.07 & 0.03 & 1.07 & 0.03 & 1.08 & 0.06 & 0.83 & \\
\hline Occipital' & 1.09 & 0.04 & 1.12 & 0.05 & 1.16 & 0.09 & 0.01 & $H<D$ \\
\hline Anterior cingulatel & 1.26 & 0.08 & 1.26 & 0.07 & 1.24 & 0.09 & 0.67 & \\
\hline Posterior cingulate' & 1.25 & 0.13 & 1.27 & 0.11 & 1.33 & 0.13 & 0.21 & \\
\hline
\end{tabular}

I. Upper slice.

*Unmedicated CFS $>$ CFS on antidepressants $(P<0.05$, $t$-test $)$

†nly non-medicated chronic fatigue syndrome (CFS) patients; one-way ANOVA $P\left(F_{2,40}\right)=0.02, F>D$.

$\mathrm{HV}$, healthy volunteers. 
(a)

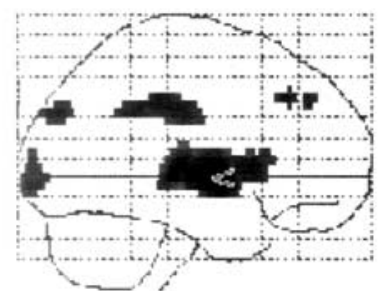

Sagittal

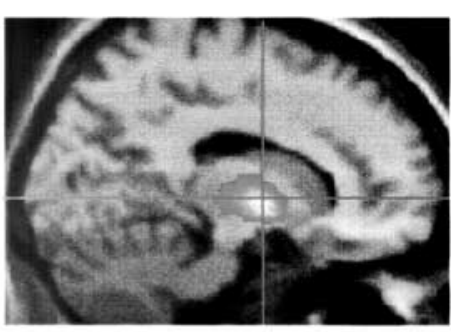

(b)

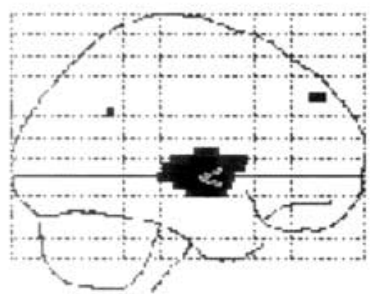

Sagittal

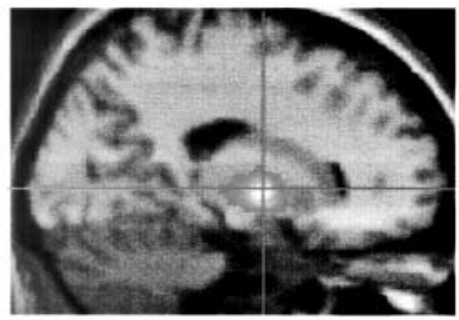

(c)

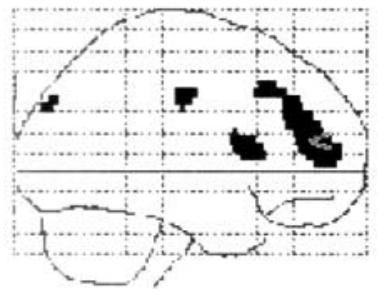

Sagittal

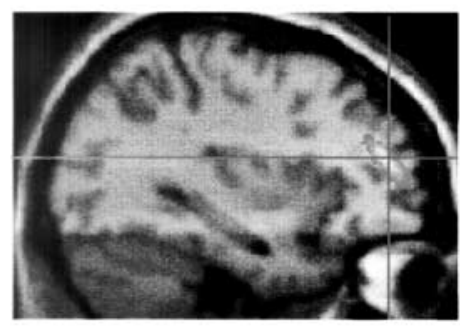

L $\quad \mathbf{R}$

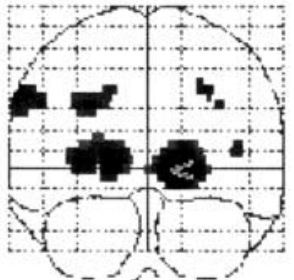

Coronal

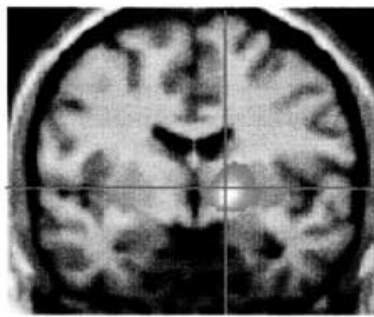

$\mathbf{R}$

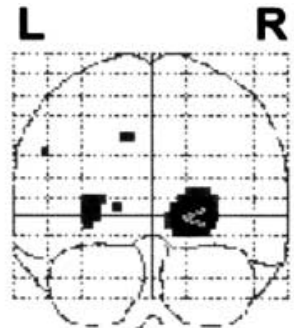

Coronal

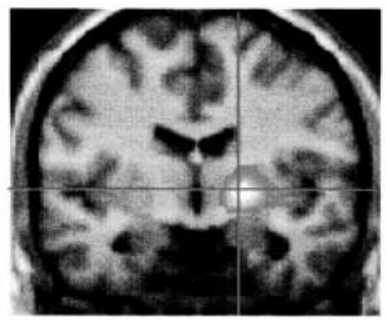

L

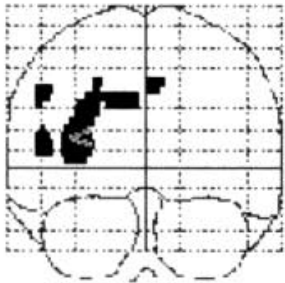

Coronal

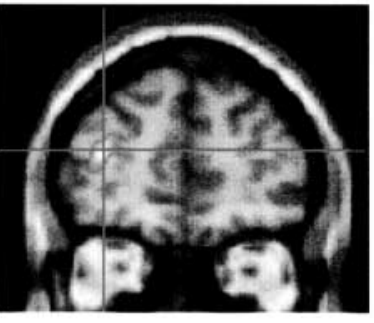

$\mathbf{R}$

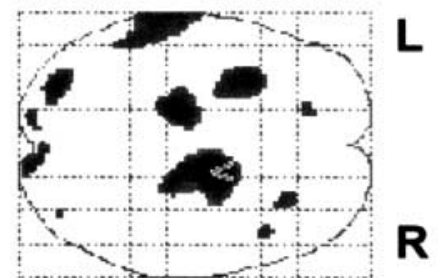

Transverse
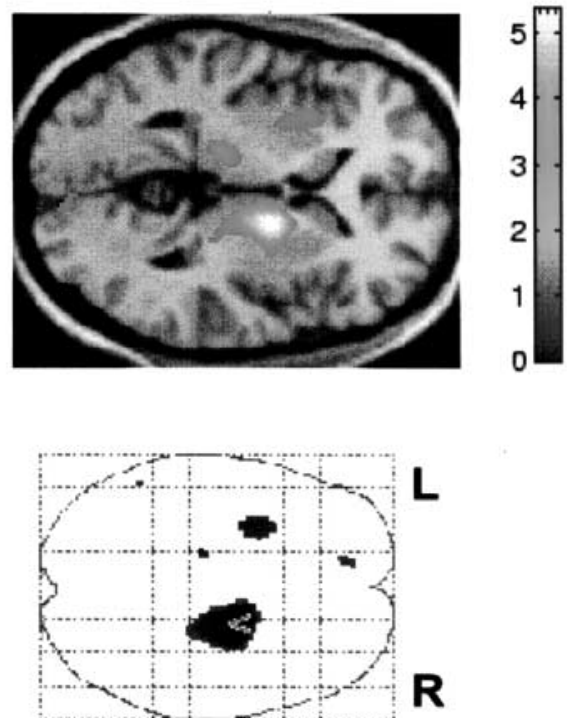

Transverse
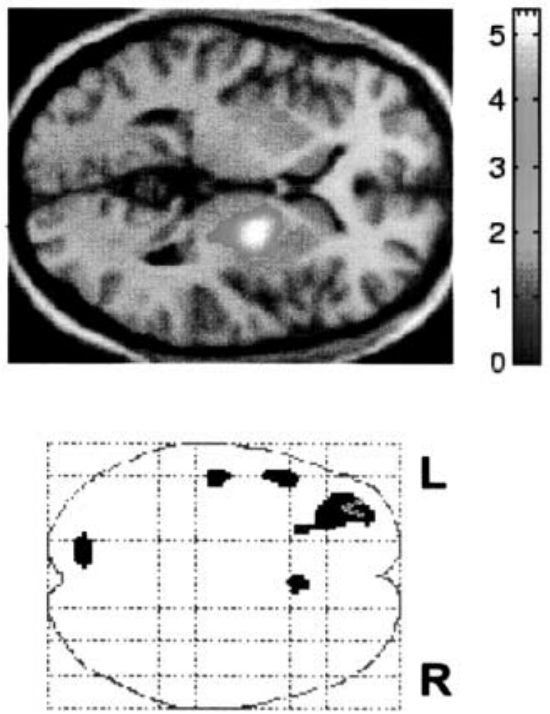

Transverse
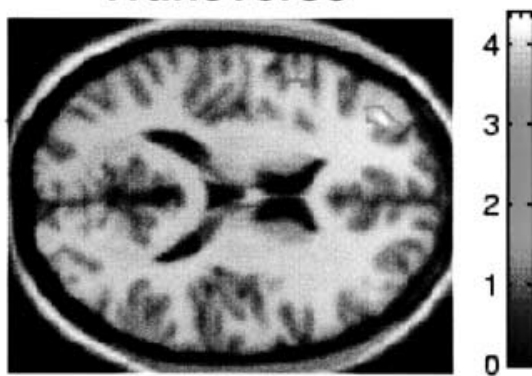

Fig. I Voxels with increased perfusion in patient groups, compared with healthy volunteers and with each other. The cross-point marks the peak difference: (a) chronic fatigue syndrome $v$. healthy volunteers; (b) depression v. healthy volunteers; (c) depression v. chronic fatigue syndrome. 
technique requires the fitting by an operator of a standard template to each individual brain. Only $20 \%$ of the volume available from the scan was utilised in the ROI analysis. SPM analysis, in contrast, uses an objective approach to analysis, with information from the whole brain, allowing for the detection of smaller effects. In particular, small structures such as the head of the caudate may be missed or captured incompletely by an ROI method that fits the whole slice template to the outer brain boundary. The central grey nuclei are particularly prone to such errors, because they lie adjacent to the lateral ventricles. Ventricular dilatation may thus appear to reduce basal ganglia perfusion, due to partial volume effects. Specifically, the reduction of perfusion in caudate ROIs in depression may be due to ventricular dilatation, while the absence of such a reduction in caudate perfusion in the more reliable SPM analysis probably reflects the absence of real tissue perfusion changes. The veracity of this interpretation can, of course, only be tested by parallel structural and functional imaging.

\section{Implications}

The relatively strong and statistically significant association of increased perfusion in the right thalamus in the subjects with CFS and depression is consistent with the clinical and biological overlap between the two disorders. Both conditions are associated with low mood and inactivity, but these are unlikely to result in increased thalamic perfusion. The similarities in disturbances of motor function (Lawrie et al, 2000) and effort perception (Lawrie et al, 1997) in CFS and depression are more likely explanations. The thalamus is generally regarded as a sensory relay station for afferent connections with the cortex. However, recent work with primates and in vivo imaging in humans increasingly suggests its involvement with motor function and planning in a system that links cerebellum and prefrontal cortex (Percheron et al, 1996). Thalamic output on the cerebral cortex is modulated in response to levels of sleep and wakefulness (Steriade \& Contreras, 1995), and recent work has demonstrated its relevance to general attention and vigilance (Kinomura et al, 1996; Roland, 1996) and to the discrimination of painful stimuli (Lenz et al, 1995). Some authors have proposed a dynamic role for the thalamus in modulating information transmission to the cortex (Sherman \& Guillery,

\section{CLINICAL IMPLICATIONS}

- The patterns of cerebral perfusion in chronic fatigue syndrome (CFS) and in depression show important similarities and differences.

Biological disturbance in CFS is not limited to those with comorbid depressive symptoms.

- These changes are quantitative rather than qualitative - single-photon emission computerised tomography has (as yet) no role in the clinical diagnosis of CFS.

\section{LIMITATIONS}

- Many of the patients were on medication.

- A relatively small number of patients with depression was included.

- The statistical parametric mapping and region of interest results are not entirely consistent.

SIOBHAN M. MACHALE, MRCPsych, STEPHEN M. LAWRIE, MRCPsych, JONATHAN T. O. CAVANAGH, MRCPsych, MIKE F. GLABUS, PhD, CATHERINE L. MURRAY, BSc, KLAUS P. EBMEIER, MRCPsych, University Department of Psychiatry and MRC Brain Metabolism Unit, Royal Edinburgh Hospital, Edinburgh; GUY M. GOODWIN, MRCPsych, University Department of Psychiatry, Warneford Hospital, Oxford

Correspondence: Professor K. P. Ebmeier, Department of Psychiatry, Edinburgh University, Royal Edinburgh Hospital, Morningside Park, Edinburgh EHIO 5HF, UK

(First received 5 July 1999, final revision 4 November 1999, accepted 10 November 1999)

1996). Others have suggested a crucial role for the thalamus in the modulation of motor and cognitive coordination (Roland, 1996). Importantly for CFS, the lateral ventral nucleus of the thalamus receives input from muscle afferents and the cerebellum that provide critical information about motor state. There is an intriguing overlap between these sensorimotor functions and the core symptoms reported in CFS. Thalamic overactivity in CFS (and depression) may, therefore, reflect increased attention to motor and cognitive tasks, with previously automatic tasks requiring higher levels of vigilance and thereby becoming effortful. Many similar disturbances and symptoms are evident in depression, but we found that prefrontal perfusion distinguishes the disorders. This is in keeping with our findings, in the same group of patients, that patients with depression have similar motor but more profound cognitive deficits than patients with CFS (Lawrie et al, 2000). These important similarities and differences in the patterns of cerebral perfusion between patients with depression and those with CFS may, therefore, be associated with their similar but different clinical presentations.

\section{ACKNOWLEDGEMENTS}

We thank the Scottish Home and Health Department and the Wellcome Trust for financial support, M. van Beck, N. Dougall and C. Sidey for expert clinical and technical support, and Norma Brearley for the careful preparation of the manuscript. We are also grateful to all the research participants, without whom this study would not have been possible.

\section{REFERENCES}

American Psychiatric Association (1987) Diagnostic and Statistical Manual of Mental Disorders (3rd edn, revised) (DSM-III-R). Washington, DC: American Psychiatric Association.

Chalder, T., Berelowitz, G., Pawlikowska, T., et al (1993) Development of a fatigue scale. Journal of Psychosomatic Research, 37, 147-153.

Costa, D. C., Tannock, C. \& Brostoff, J. (1995) Brainstem perfusion is impaired in chronic fatigue syndrome. Quarterly Journal of Medicine, 88, 767-773. 
Ebmeier, K. P., Dougall, N. J., Austin, M.-P., et al (1991) The split-dose technique for the study of psychological and pharmacological activation with the cerebral blood flow marker exametazime and single photon emission computed tomography (SPECT): reproducibility and rater reliability. International Journal of Methods in Psychiatric Research, I, 27-38.

\section{—, Steele, J. D., MacKenzie, D. M., et al (1995)}

Cognitive brain potentials and regional cerebral blood flow equivalents during two- and three-sound auditory "oddball tasks". Electroencephalography \& Clinical Neurophysiology, 95, 434-443.

_ , Cavanagh, J. T. O., Moffoot, A. P. R., et al (1997) Cerebral perfusion correlates of depressed mood. British Journal of Psychiatry, 170, 77-8I.

Endicott, J. \& Spitzer, R. L. (1978) A diagnostic interview - the schedule for affective disorders and schizophrenia. Archives of General Psychiatry, 35, 837-844.

Fischler, B., D'Haenen, H., Cluydts, R., et al (1996) Comparison of ${ }^{99 \mathrm{mT}} \mathrm{c}$ HMPAO SPECT scan between chronic fatigue syndrome, major depression and healthy controls: an exploratory study of clinical correlates of regional cerebral blood flow. Neuropsychobiology, 34, 175-183.

Friston, K. J., Holmes, A. P., Worsley, K. J., et al (1995) Statistical parametric maps in functional imaging: a genera linear approach. Human Brain Mapping, 2, 189-210.

Fukuda, K., Straus, S., Hickie, l., et al (1994) The chronic fatigue syndrome: approach to its definition and study. Annals of Internal Medicine, I2I, 953-959.

Goldstein, J. A., Mena, I., Jouanne, E., et al (1995) The assessment of vascular abnormalities in late life chronic fatigue syndrome by brain SPECT: comparison with late life major depressive disorder. Journal of Chronic Fatigue Syndrome, I, 55-79.
Goodwin, G. M. (1997) Neuropsychological and neuroimaging evidence for the involvement of the frontal lobes in depression. Journal of Psychopharmacology, II, II5-122.

Hamilton, M. (1960) Rating Scale for Depression Journal of Neurology, Neurosurgery and Psychiatry, 23 $56-62$.

Ichise, M., Salit, I. E., Abbey, S. E., et al (1992) Assessment of regional cerebral perfusion by ${ }^{99} \mathrm{Tc}^{\mathrm{m}}$ HMPAO SPECT in chronic fatigue syndrome. Nuclear Medicine Communications, 13, 767-772.

Kendell, R. E. (1991) Chronic fatigue, viruses, and depression. Lancet, 337, 160-162.

Kinomura, S., Larsson, J., Gulyás, B., et al (1996) Activation by attention of the human reticular formation and thalamic intralaminar nuclei. Science, 27I, 512-515.

Lawrie, S. M., MacHale, S. M., Power, M. J., et a (1997) Is the chronic fatigue syndrome best understood as a primary disturbance of the sense of effort? Psychological Medicine, 27, 959-999.

_, _, Cavanagh, J.T. O., et al (2000) The difference in patterns of motor and cognitive function in chronic fatigue syndrome and severe depressive illness. Psychological Medicine (in press).

Lenz, F. A., Gracely, R. H., Romanoski, A. J., et a (1995) Stimulation in the human somatosensory thalamus can reproduce both the affective and sensory dimensions of previously experienced pain. Nature Medicine, I, 910-913.

Nelson, H. E. (1982) National Adult Reading Test for the Assessment of Premorbid Intelligence in Patients with Dementia: Test Manual.Windsor: NFER-Nelson.

Percheron, G., Francois, C., Talbi, B., et al (1996) The primate motor thalamus. Brain Research Reviews, 22. 93-|8|.
Peterson, P. K., Sirr, S. A., Grammith, F. C., et a (1994) Effects of mild exercise on cytokines and cerebral blood flow in chronic fatigue syndrome patients. Clinical and Diagnostic Laboratory Immunology, I, 222-226.

Roland, P. E. (1996) The system for regulating general attention in the human brain. Molecular Psychiatry, $\mathbf{I}$ 303-304.

Schwartz, R. B., Komaroff, A. L., Garada, B. M., et al (1994) SPECT imaging of the brain: Comparison of findings in patients with chronic fatigue syndrome, AIDS dementia complex, and major unipolar depression. American Journal of Roentgenology, 162, 943-95I.

Sherman, S. M. \& Guillery, R.W. (1996) Functiona organization of thalamocortical relays. Journal of Neurophysiology, 76, 1367-1395.

Steriade, M. \& Contreras, D. (1995) Relations between cortical and thalamic cellular events during transition from sleep patterns to paroxysmal activity. Journal of Neuroscience, 15, 623-642.

Szikla, G., Bouvier, G., Hori, T., et al (1977)

Angiography of the Human Brain Cortex. New York: Springer.

Talairach, J., Zilkha, G., Tournoux, P., et al (1988) Atlas d'Anatomie stéréotactique du Télencéphale. Paris: Masson

von Zerssen, D., Strian, F. \& Schwarz, D. (1974) Evaluation of depressive states, especially in longitudinal studies. In Psychological Measurements in Psychopharmacology (ed. P. Pichot). Paris: Karger

Zigmond, A. S. \& Snaith, R. P. (1983) The Hospital Anxiety and Depression Scale. Acta Psychiatrica Scandinavica, 67, 361-370 